\title{
THE KRULL-SCHMIDT THEOREM FOR INTEGRAL GROUP REPRESENTATIONS
}

\author{
BY IRVING REINER ${ }^{1}$ \\ Communicated by Paul T. Bateman, March 13, 1961
}

Let $R_{0}$ be the ring of algebraic integers in an algebraic number field $K$, let $P$ be a prime ideal in $R_{0}$, and let $R_{P}$ (or briefly $R$ ) denote the ring of $P$-integral elements of $K$. Choose $\pi \in R_{0}$ such that $\pi R$ is the unique maximal ideal in $R$. Further let $K^{*}$ be the $P$-adic completion of $K$, with ring of $P$-adic integers $R^{*}$. For a fixed finite group $G$, we understand by the term " $R_{0} G$-module" a left $R_{0} G$-module which as $R_{0}$-module is torsion-free and finitely-generated; analogous definitions hold for $R G$ - and $R^{*} G$-modules.

Swan $[9 ; 10]$ has recently proved that the Krull-Schmidt theorem is valid for projective $R^{*} G$-modules. We show here the following main result, which is a consequence of some work of Maranda $[3 ; \mathbf{4}]$ :

THEOREM 1. The Krull-Schmidt theorem holds for arbitrary $R^{*} G$ modules, that is, if $M_{1}, \cdots, M_{r}, N_{1}, \cdots, N_{s}$ are indecomposable $R^{*} G$-modules such that

$$
M_{1}+\cdots+M_{r} \cong N_{1}+\cdots+N_{s}
$$

(the notation indicating external direct sums), then $r=s$, and after renumbering the $\left\{N_{j}\right\}$ if need be, $M_{1} \cong N_{1}, \cdots, M_{r} \cong N_{r}$.

To prove this and some corollaries we make use of the following results of Maranda $[3 ; 4]$.

(i) Let $M$ and $N$ be $R^{*} G$-modules, and let $e$ be the largest integer for which $P^{e}$ divides the order of $G$. If $M \cong N$ then

$$
M / \pi^{d} M \cong N / \pi^{d} N
$$

as $\left(R^{*} / \pi^{d} R^{*}\right) G$-modules

for all $d$.

Conversely if (2) holds for some $d>e$, then $M \cong N$. Furthermore, the same result holds for $R G$-modules.

(ii) Let $M$ and $N$ be $R G$-modules. Then $M \cong N$ if and only if $R^{*} M \cong R^{*} N$.

(iii) Let $M$ be an $R^{*} G$-module. If $M$ is decomposable, so is $M / \pi^{d} M$ for all $d$. Conversely if $M / \pi^{d} M$ is decomposable as $\left(R^{*} / \pi^{d} R^{*}\right) G$ module for some $d>2 e$, then $M$ is also decomposable.

${ }^{1}$ This research was supported in part by the Office of Naval Research. The author wishes to thank Professor A. Heller for some helpful conversations. 
Now fix $d=2 e+1$, and let $\bar{M}=M / \pi^{d} M, \bar{R}^{*}=R^{*} / \pi^{d} R^{*}$, and so on. If (1) holds then we have

$$
\bar{M}_{1}+\cdots+\bar{M}_{r} \cong \bar{N}_{1}+\cdots+\bar{N}_{s}
$$

as $\bar{R} G$-modules, and each of the above summands is indecomposable by virtue of (iii). But $\bar{R}^{*} G$ is a ring with minimum condition, and so the Krull-Schmidt theorem is valid for $\bar{R}^{*} G$-modules (see [2]). Therefore $r=s$, and renumbering the $\left\{\bar{N}_{j}\right\}$ if need be, we have

$$
\bar{M}_{1} \cong \bar{N}_{1}, \cdots, \bar{M}_{r} \cong N_{r} .
$$

The conclusion now follows by (i).

Corollary 1. Let $L, M, N$ be $R G$-modules such that $M+L \cong N+L$. Then $M \cong N$.

Corollary 2. Let $M^{(t)}$ denote the direct sum of $t$ copies of $M$. If $M$, $N$ are $R G$-modules such that $M^{(t)} \cong N^{(t)}$ for some $t$, then $M \cong N$.

Corollary 3. The Krull-Schmidt theorem holds for indecomposable $R G$-modules which remain indecomposable in passing to $R^{*}$. In particular, it is valid for absolutely irreducible $R G$-modules. [10].

The next corollary partially answers a question raised by Swan

Corollary 4. Let $L, M, N$ be $R_{0} G$-modules such that $M \dot{+} L \cong N \dot{+} L$. Then for each $P$ we have $R_{P} M \cong R_{P} N$. (If in particular $M$ is absolutely irreducible, and $R_{0}$ has class number 1 , then from Maranda [4] we may conclude that $M \cong N$.)

It is still an open question as to whether the Krull-Schmidt theorem holds for $R G$-modules. That it fails for $R_{0} G$-modules already follows from [5], but the following approach is also instructive. Let $N$ be an $R_{0} G$-submodule of the $R_{0} G$-module $M$, such that $K N=K M$, and define

$$
\operatorname{ann}(M / N)=\left\{\alpha \in R_{0}: \alpha M \subset N\right\} .
$$

Then we have

THEOREM 2. Let $N_{1}, N_{2}$ be submodules of the $R_{0} G$-module $M$ such that $K N_{1}=K N_{2}=K M$, and suppose that

$$
\operatorname{ann}\left(M / N_{1}\right)+\operatorname{ann}\left(M / N_{2}\right)=R .
$$

Then $N_{1}+N_{2} \cong M+\left(N_{1} \cap N_{2}\right)$.

Proof. Choose $\alpha_{i} \in$ ann $\left(M / N_{i}\right), i=1,2$, so that $\alpha_{1}+\alpha_{2}=1$. Then 
$\left(n_{1}, n_{2}\right) \rightarrow\left(n_{1}+n_{2}, \alpha_{2} n_{1}-\alpha_{1} n_{2}\right)$ gives the desired isomorphism.

In particular let $M$ be absolutely irreducible, and let $C$ denote an ideal in $R_{0}$. From [4] or [6] it follows that $M \cong C M$ if and only if $C$ is principal. Hence if $R_{0}$ has class number $>1$, and if $A, B$ are nonprincipal ideals of $R_{0}$ such that $A+B=R$, then we have from the above

$$
A M+B M \cong M+A B M,
$$

which shows that the Krull-Schmidt theorem does not hold.

Using a result of D. G. Higman's [1] (see also [6]) one can show that Theorems 1 and 2 are still valid when $R_{0} G$ is replaced by an $R_{0}$-order in a separable $K$-algebra, and likewise with $R$ or $R^{*}$ in place of $R_{0}$.

Related problems are studied in $[7 ; 8 ; 11]$.

Added in proof. The author has recently discovered that Theorem 1 has been proved previously by R. G. Swan [unpublished] and also by Borevich and Faddeyev [12], by a different approach. The corollaries and Theorem 2 are new, however.

\section{BIBLIOGRAPHY}

1. D. G. Higman, On orders in separable algebras, Canad. J. Math. vol. 7 (1955) pp. 509-515.

2. N. Jacobson, The theory of rings, Mathematical Surveys, no. 1, American Mathematical Society, 1943.

3. J.-M. Maranda, On P-adic integral representations of finite groups, Canad. J. Math. vol. 5 (1953) pp. 344-355.

4. - On the equivalence of representations of finite groups by groups of automorphisms of modules over Dedekind rings, Canad. J. Math. vol. 7 (1955) pp. 516-526.

5. I. Reiner, Integral representations of cyclic groups of prime order, Proc. Amer. Math. Soc. vol. 8 (1957) pp. 142-146.

6. - On the class number of representations of an order, Canad. J. Math. vol. 11 (1959) pp. 660-672.

7. - The non-uniqueness of irreducible constituents of integral group representations, Proc. Amer. Math. Soc. vol. 11 (1960) pp. 655-657.

8. I. Reiner and H. Zassenhaus, Equivalence of representations under extensions of local ground rings, Illinois J. Math. vol. 5 (1961) (will appear in September 1961).

9. R. G. Swan, Projective modules over finite groups, Bull. Amer. Math. Soc. vol. 65 (1959) pp. 365-367.

10. — Induced representations and projective modules, mimeographed notes, University of Chicago, 1959.

11. S. Takahashi, Arithmetic of group representations, Tôhoku Math. J. vol. 11 (1959) pp. 216-246.

12. Z. I. Borevich and D. K. Faddeyev, Theory of homology in groups. II, Proc. Leningrad Univ. vol. 7 (1959) pp. 72-87.

UNIVERSITY OF ILLINOIS 